\title{
Ethylmalonic encephalopathy associated with respiratory failure
}

\author{
Halil Köse ${ }^{1, *}$, Mehmet Hamdi Şahan ${ }^{2}$, Halil İbrahim Aydin ${ }^{3}$ \\ 1 Şanliurfa Pediatric Diseases Hospital, Pediatric Intensive Care Unit, Şanlunrfa, Turkey \\ ${ }^{2}$ Kızılaslan Medical Center, Department of Radiology, Gaziantep, Turkey \\ ${ }^{3}$ Turgut Özal University Faculty of Medicine, Department of Pediatric Metabolism Diseases, Ankara, Turkey
}

\begin{abstract}
Ethylmalonic encephalopathy (EE) is a rarely encountered metabolic disease with autosomal recessive inheritance and is characterized by development retardation, acrocyanosis, petechiae and chronic diarrhea. In the literature, EE has usually presented itself with neurological disorders, developmental retardation, acrocyanosis and skin lesions. We encountered no case in the literature accompanied by respiratory failure. EE should be considered in the patients with severe respiratory failure, exanthematous skin lesion, acidosis, chronic diarrhea, neurological symptoms and pathological signal increment in the basal ganglia on MRI together with the history of sibling death and consanguineous marriage.

Herein, a 2.5-year-old boy case, who presented with exanthematous lesions over the body and respiratory failure and had lactic acidemia, increase in serum C4 and C5 acylcarnitine derivatives, excretion of ethylmalonic acid, methyl succinic acid, isovalerylglycine and isobutyrylglycine in the urine, pathological signal increment in the basal ganglia on CT and MRI, and firstly defined homozygote deletion in the ETHE1 gene in genetic analysis.

In conclusion, we intended to emphasize consider underlying EE metabolic disease in the cases with frequent lower respiratory tract infection and respiratory failure in the presence of hypotonia and neuromotor developmental retardation.
\end{abstract}

Key Words: Brain diseases, metabolic, multiple acyl coenzyme a dehydrogenase deficiency, respiratory insufficiency

\section{Introduction}

Exanthematous infectious diseases that involve respiratory system are considered at first in the patients that present with respiratory failure and exanthematous skin lesions. However, neurological and metabolic diseases should be considered in hypotonic patients with acidosis, who have history of neurological development delay, undefined death of a sibling with similar features and consanguineous marriage. Lactic acidemia is defined as increased acylcarnitine concentration in serum and ethylmalonic acid and ethylmalonic acid derivatives in the urine and ethylmalonic aciduria. Ethylmalonic aciduria is certainly accompanied by encephalopathy $(1,2)$.

EE, which shows autosomal recessive inheritance, has been demonstrated in animal experiments that inhibited mitochondrial kinase activity, although its underlying mechanism is undefined. Considering the importance of creatinine kinase for brain energy balance may explain neurological features of EE observed in such patients (3). EErelated symptoms emerge from decreased energy production in cells and deposition of toxic metabolites in the body. Pathological signal increment is observed in the basal ganglia, periventricular white matter and cerebellar dentate nuclei on MRI (4). In the present case report, we intended to introduce respiratory failure presentation of an EE case, which is a rarely encountered metabolic disease, by means of clinical, laboratory and radiological features.

\section{Case report}

A 2.5-year-old boy patient was brought to our polyclinic with coughing, fever, difficulty in breathing and rashes over his body. Fever, coughing and rapid breathing complaints have existed for two days. Due to worsening in tachypnea and acidotic breathing in the form of sighing during a few hours of monitoring in the emergency room, he was admitted to the pediatric intensive care unit; he was intubated and mechanical ventilator support was provided (Photo 1,2,3). Medical history of the patient revealed that he has been given birth via normal vaginal delivery at term, delivery was difficult, mental and motor development progressed behind age, has had frequent lower respiratory tract infection and has had vomiting and diarrhea at 
intervals. His family history revealed that his parents were distant relatives. The patient had four healthy siblings. His brother, who deceased at the age of 4.5 years without being diagnosed, had motor-mental retardation and skin lesions similar to those found in our patient. History of deceased brother revealed that he was unable to speak or walk and had developmental retardation. Again, he had symptoms similar to the skin lesions found in our patient.

On the physical examination of the patient at admission, his general status was poor, he was hypotonic, confused and had widespread petechial and purpuric exanthematous lesions, which do not blanche on finger pressure and partially tend to coalesce. He had acrocyanosis in the extremities. $\mathrm{He}$ had tachypnea and acidotic breathing. His percentile scores were as following: body weight: $8900 \mathrm{gr}(<3 \mathrm{P})$, height: $79 \mathrm{~cm}(3-10 \mathrm{P})$ and head circumference: $47 \mathrm{~cm} \mathrm{(3-10} \mathrm{P).} \mathrm{On} \mathrm{his} \mathrm{laboratory}$ analyses, blood gas analyses showed metabolic and respiratory acidosis; there were leukocytosis
$(27.100 / \mathrm{mL})$ and thrombocytosis $\left(544000 / \mathrm{mm}^{3}\right)$; peripheral blood smear analysis and bone marrow revealed no atypical cells. Among coagulation tests, PT and aPTT values were normal. Lactate concentration of the patient was high as 3.6 $\mathrm{mmol} / \mathrm{L}$. Differential diagnosis from respiratory system infections and other exanthematous infectious diseases that present with rashes was performed both clinically and by laboratory analyses. Pathological signals were observed in the bilateral basal ganglia, lentiform and caudate nuclei on cranial CT and MRI examination; hypodense nodular areas were observed on multifocal CT (Figure 1); and hypointense pathological signals on T1-weighted sequences and hyperintense pathological signals on T2weighted sequences were observed on MRI (Figure 2,3). Contrast-enhanced examination demonstrated contrast agent uptake in the defined areas, and contrasting in gyral pattern attracted attention in both frontal regions.

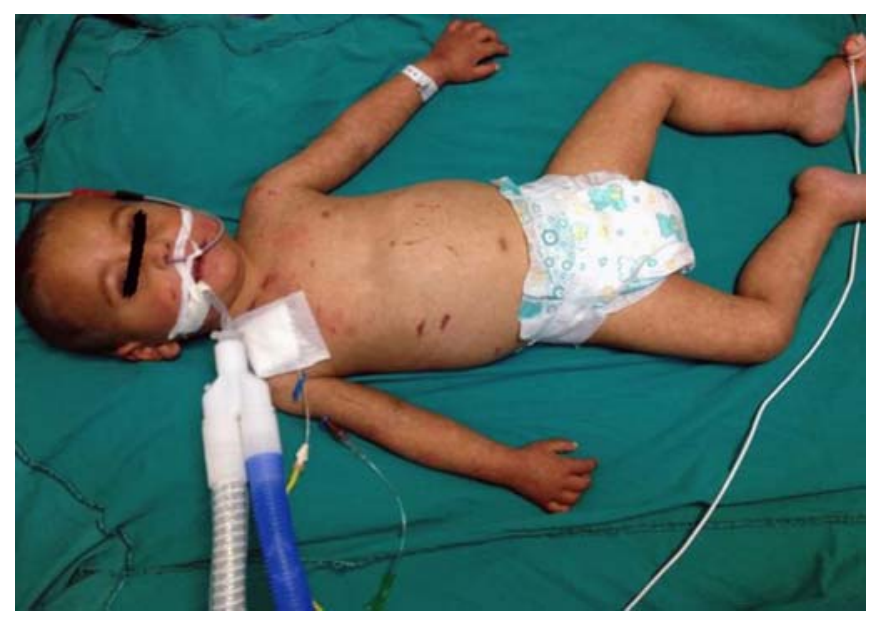

Photo 1. In the body, petechiae, rash lesions and mechanical ventilatory support was provided due to respiratory failure.
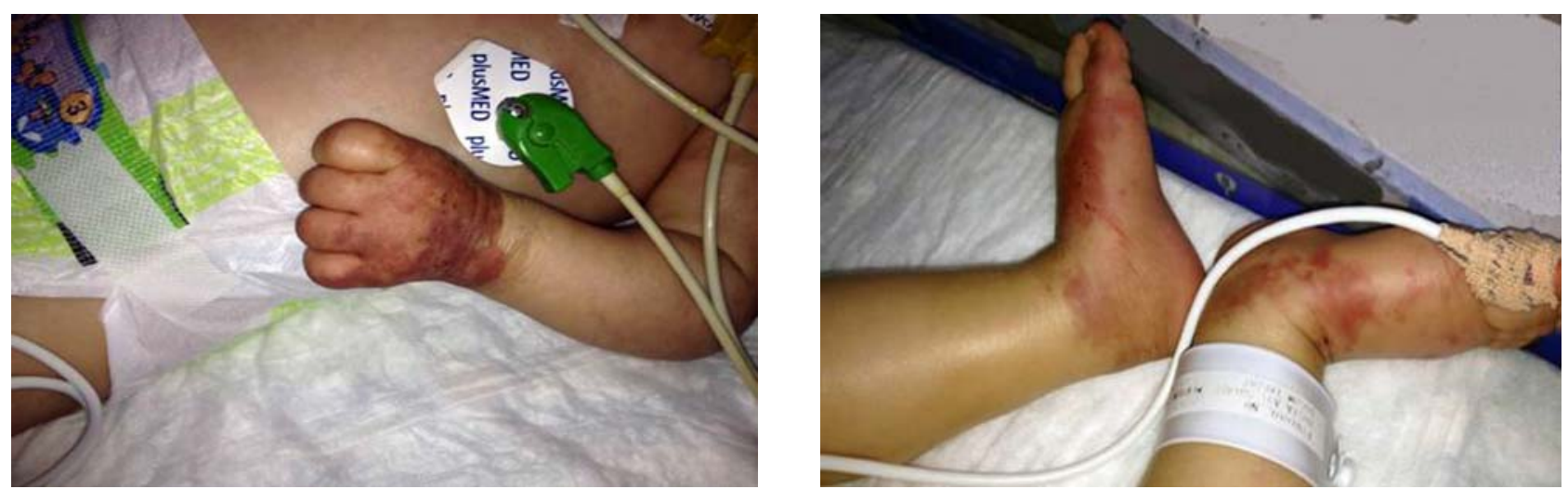

Photo 2, 3. Extremities, acrocyanosis and petechiae.

East J Med Volume:21, Number:4, October-December/2016 
Blood carnitine-acylcarnitine analysis revealed that free carnitine was within the normal ranges (47.19 $\mu / \mathrm{L})$, whereas increase was determined in C4butyrylcarnitine $(5.9 \mu / \mathrm{L}$; normal: <1.1), C5isovalerylcarnitine $(1.25 \mu / \mathrm{L}$; normal: <0.47), C5$\mathrm{OH} \quad(0.86 \mu$;normal: $<0.69) \quad$ and $\quad$ C6hexanoylcarnitine $(3.57 \mu / \mathrm{L}$; normal: <0.24). In the urine organic acid analysis, we determined $1440 \mathrm{mmol} / \mathrm{mol}$ creatinine ethylmalonic acid, 60 $\mathrm{mmol} / \mathrm{mol}$ creatinine isovalerylglycine, 60 $\mathrm{mmol} / \mathrm{mol}$ creatinine N-butyrylglycine, 60 $\mathrm{mmol} / \mathrm{mol}$ creatinine isobutyrylglycine, 60 $\mathrm{mmol} / \mathrm{mol}$ creatinine suberic acid, and 12 $\mathrm{mmol} / \mathrm{mol} \quad$ creatinine hexanoylglycine excretion. The patient had homozygote and his

parents had heterozygote deletion in the exons 5, 6 and 7 in the ETHE1 gene. The deletion determined in the patient has been defined for the first time.

\section{Discussion}

$\mathrm{EE}$ is an autosomal recessive disease caused by ETHE1 gene (chromosome 19q13) mutations and manifests itself with nervous system, gastrointestinal system and blood vessel abnormalities $(1,5)$. Genetic testing is of great importance since a healthy "carrier" couple with mutation in a single allele in ETHE1 gene has a risk by $25 \%$ in each pregnancy $(2,5)$.

It is characterized by persistent lactic acidemia, increase in C4 and C5 acylcarnitine derivatives and excretion of ethylmalonic acid, methylsuccinic acid, isovalerylglycine and isobutyrylglycine in the urine. Lactic acidosis becomes prominent with intervening infections (2,6). It has been propounded that ETHE 1 protein shows sulphur dioxygenase activity and plays a role in mitochondrial metabolic pathway that is responsible for sulphide detoxification $(2,5)$.

Underlying metabolic defect is not known definitely. Whether it is associated with the metabolisms of isoleucine, methionine or other amino acids is not clear $(7,8)$. EE has been

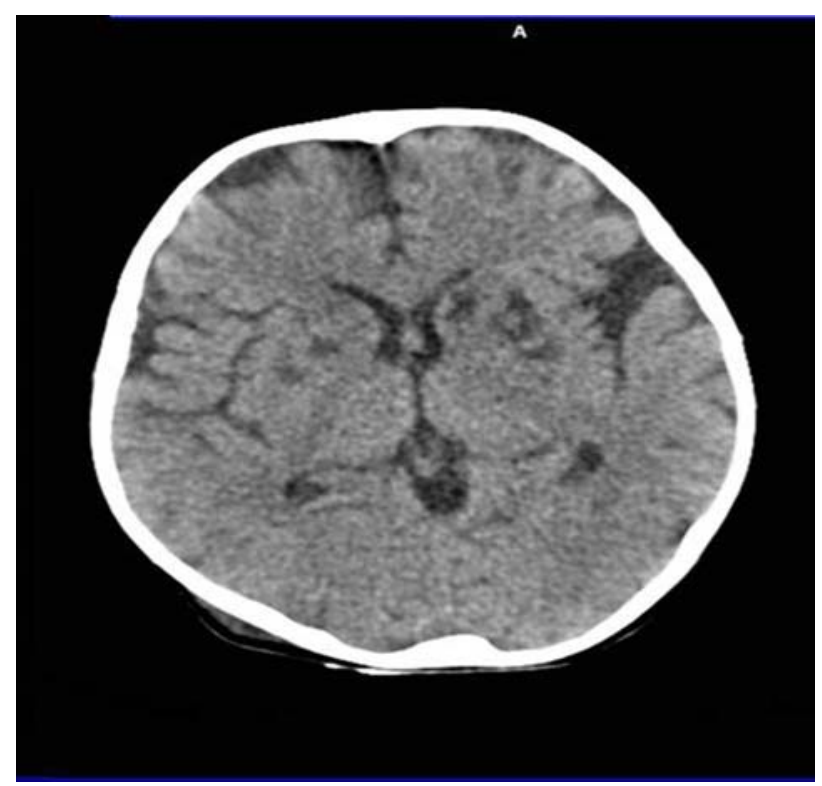

Fig. 1. In CT; hypodense nodular areas were observed on multi-focal.
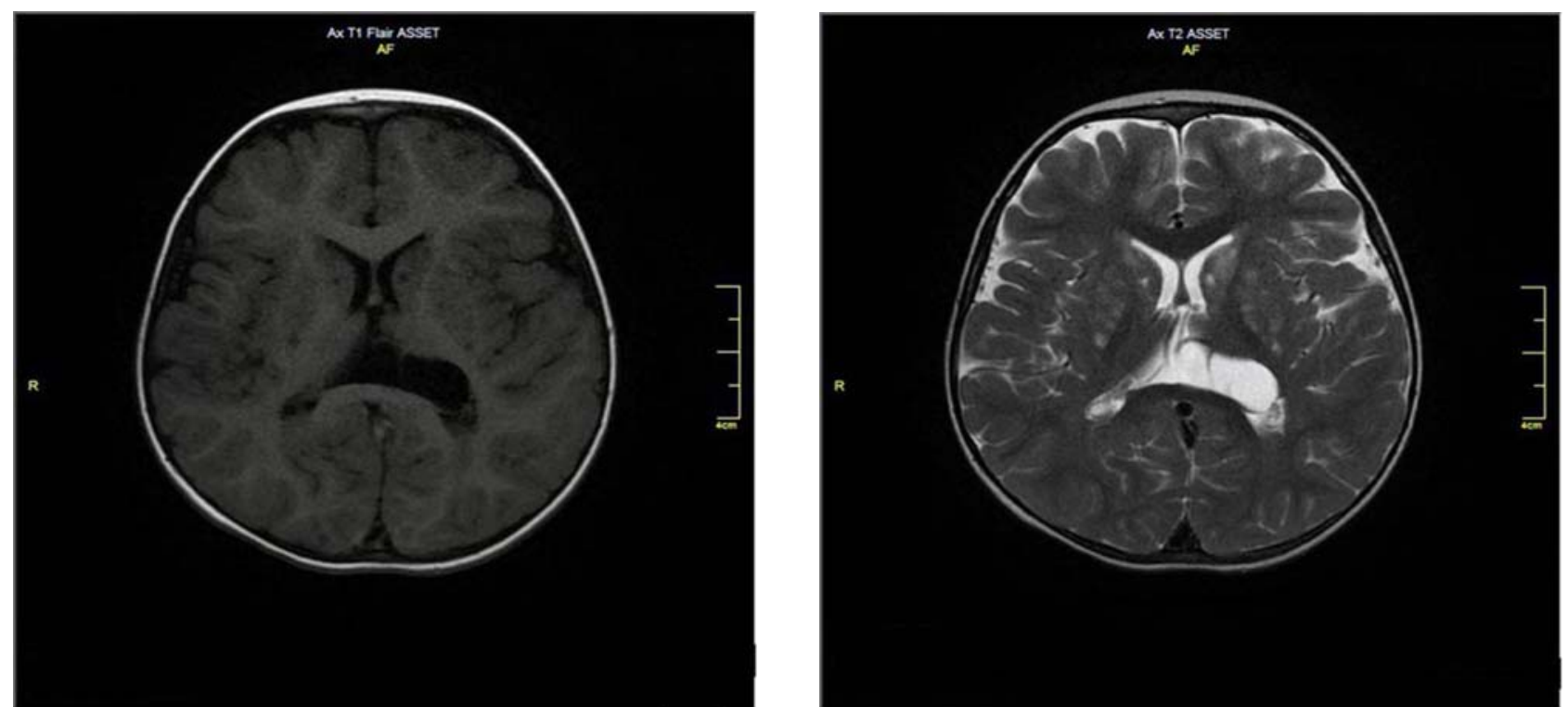

Fig. 2, 3. In MRI; hypointense pathological signals on T1-weighted sequences and hyperintense pathological signals on T2-weighted sequences were observed. 
demonstrated in animal experiments that inhibited mitochondrial creatinine kinase activity in skeletal muscle, heart and brain. Consideration of the importance of creatinine kinase for cerebral energy balance may explain neurological features observed in EE in such patients (3). On MRI, symmetric infarction areas can be observed in the basal ganglia (caudate and putamen), periventricular white matter and cerebellar dentate nuclei (4). EE-related symptoms arise from decreased energy production in the cells and deposition of toxic metabolites in the body $(5,8)$.

The symptoms of EE usually become apparent at birth or in the first few months of life and progressively become worse. The symptoms include developmental retardation, hypotonia, abnormal movements in the arms and legs, seizures, petechiae, cyanosis, acrocyanosis (blue color in hands and feet due to low blood oxygen concentration), and chronic diarrhea $(1,2,5)$.

The present case had developmental retardation, hypotonia, exanthematous skin lesions, acrocyanosis and chronic diarrhea. He had the history of deceased sibling with similar clinical features. Development retardation, hypotonia and lactic acidosis in the present case, who has had frequent respiratory tract infection, have occurred probably due to accompanying related infections. Primarily the clinical and laboratory findings of the patient, of whom the main presenting symptom was respiratory failure, were investigated for exanthematous infections that involve respiratory system and differential diagnosis was made. We encountered no case with prominent respiratory symptoms in the literature; however, patients die of unexpected intervening infections, apnea and metabolic decompensation or due to respiratory failure $(2,9)$.

There is no treatment for ethylmalonic encephalopathy. Different kinds of therapies including vitamin B1, B2, B6, B12, C, E, Biotin, coenzyme Q10, low-protein diet and carnitine have been attempted, but none of them showed efficacy or benefit in the long term. Symptomoriented treatment is applied $(9,10)$. Individuals with ethylmalonic encephalopathy usually lose their life before the age of 10 years due to intervening infections (9).

In conclusion, EE should be considered in the patients with developmental retardation, history of presence of similar disease in the family or deceased sibling and consanguineous marriage together with exanthematous skin lesions, lactic acidosis, chronic diarrhea, neurological symptoms and pathological findings in basal ganglia on MRI. We intended to emphasize considering underlying EE metabolic disease in the cases with frequent lower respiratory tract infection and respiratory failure in the presence of hypotonia and neuromotor developmental retardation.

\section{References}

1. Zafeiriou DI, Augoustides-Savvopoulou P, Haas $\mathrm{D}$, et al. Ethylmalonic encephalopathy: clinical and biochemical observations. Neuropediatrics 2007; 38: 78-82.

2. Dweikat I1, Naser E, Damsah N, Libdeh BA, Bakri I. Ethylmalonic encephalopathy associated with crescentic glomerulonephritis. Metabolic Brain Disease 2012; 27: 613-616.

3. Leipnitz G, Schuck PF, Ribeiro CAJ, et al. Ethylmalonic acid inhibits mitochondrial creatine kinase activity from cerebral cortex of young rats in vitro. Neurochem Res 2003; 28: 771-777.

4. Ozano PT, Rashed M. Millington DS, et al. Ethyl malonic aciduria: an organic academia with CNS development and vasculopathy. Brain Dev 1994; 16: 12-22.

5. Tiranti V, Briem E, Lamantea E, et al. ETHE1 mutations are specific to ethylmalonic encephalopathy. J Med Genet 2006; 43: 340-346.

6. Di Rocco M, Caruso U, Briem E, et al. A case of ethylmalonic encephalopathy with atypical clinical and biochemical presentation. Mol Genet Metab 2006; 89: 395-397.

7. Nowackzyk MJM, Lehotay DC, Platt BA, et al. Ethylmalonic and methylsuccinic aciduria in ethylmalonic encephalopathy arise from abnormal isoleucine metabolism. Metabolism 1998; 47: 836839.

8. McGown KA, Nyhan W, Barshop BA, et al. The role of methionine in ethylmalonic encephalopathy with petechiae. Arch Neurol 2004; 61: 570-574.

9. Yoon HR, Hahn SH, Ahn YM, et al. Therapeutic trial in the first three Asian cases of ethylmalonic encephalopathy: Response to riboflavin. J Inherit Metab Dis 2001; 24: 870-873.

10. Heberle LC, Al Tawari AA, Ramadan DG, Ibrahim JK. Ethylmalonic encephalopathy- report of two cases. Brain Dev 2006; 28: 329-331. 